Inra Prod. Anim., 2009, 22 (3), 245-254

\title{
Alternatives aux traitements anthelminthiques en élevage biologique des ruminants
}

H. HOSTE $1,2,3$, J. CABARET ${ }^{4}$, G. GROSMOND 5 , J.-P. GUITARD 6

${ }^{1}$ INRA, UMR1225, Interactions Hôtes-Agents Pathogènes, F-31076 Toulouse, France

2 Université de Toulouse, Interactions Hôtes-Agents Pathogènes, F-31076 Toulouse, France

${ }^{3}$ ENVT, Interactions Hôtes-Agents Pathogènes, F-31076 Toulouse, France

${ }^{4}$ INRA, UR1282 Infectiologie Animale et Santé Publique, F-37380 Nouzilly, France

5 Société Hippolab, rue du Thuy, F-63290 Limons, France

${ }^{6}$ Lycée Agricole de Saint-Affrique, F-12000 Saint-Affrique, France

Courriel:h.hoste@envt.fr

De manière générale, la gestion des pathologies dans les populations animales (ou humaines) repose sur trois principes de lutte, auxquels correspondent des moyens d'action diversifiés, dont l'importance et les priorités s'agencent en fonction des agents pathogènes en cause et des moyens disponibles, sans qu'existe d'exclusive. Ces mêmes principes sont à la base des schémas de lutte contre les parasitoses des principales espèces domestiques de rente (figure 1).

1 - EVITER les contacts entre hôte et agents pathogènes ; ce qui peut être obtenu soit par réduction de la contami-

Figure 1. Les trois principaux axes de lutte contre les parasitoses et les méthodes alternatives correspondantes.

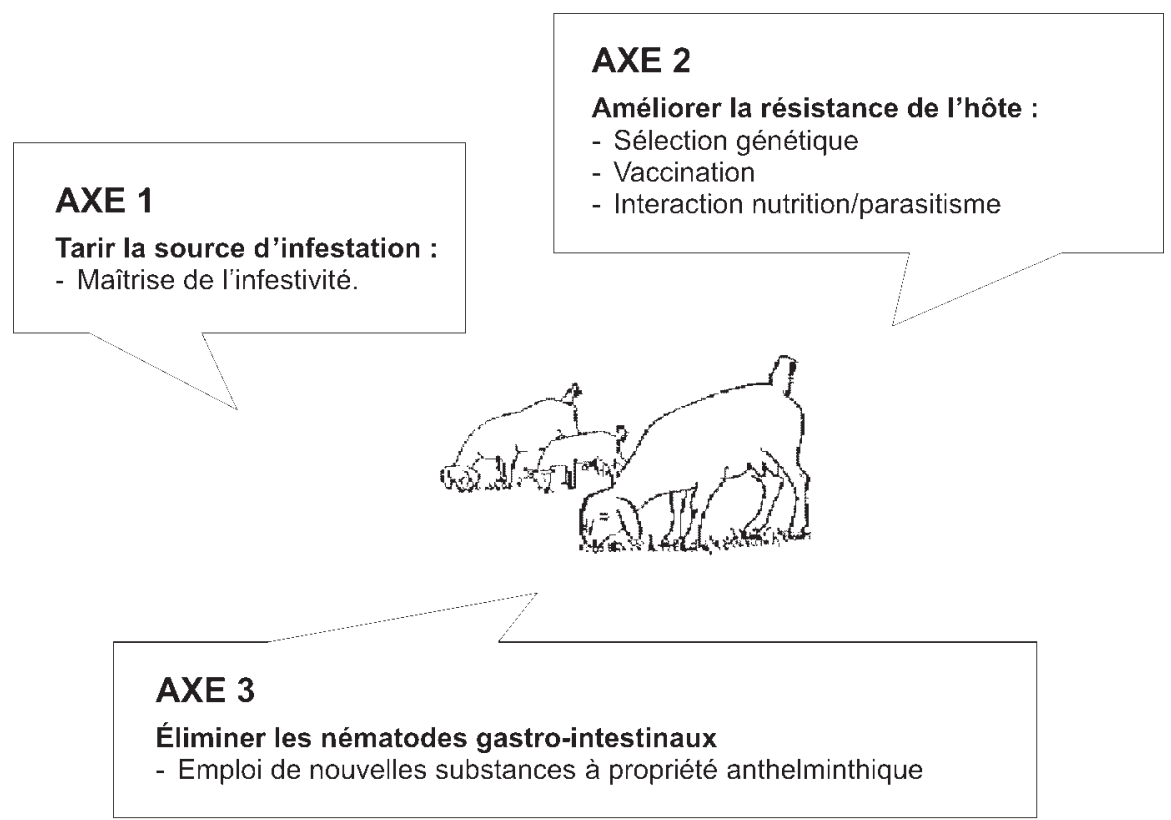

nation du milieu extérieur, soit par adaptation du comportement de l'hôte. Cela revient à appliquer les principes de l'hygiène ou de la prophylaxie sanitaire.

2 - RESISTER en augmentant la résistance de l'hôte pour réduire sa contamination et les conséquences délétères des infections. Les méthodes de prophylaxie médicale se retrouvent dans cette rubrique.

3 - TRAITER par l'application de molécules chimiques spécifiques pour éliminer (ou perturber la biologie) des populations d'agents pathogènes installées chez l'hôte.
A partir de 1960, l'efficacité et les avantages connexes (polyvalence, facilité d'emploi, faible coût...) des substances chimiques antiparasitaires commercialisées ont amené les élevages conventionnels à faire de ces molécules la pierre angulaire de la lutte contre les parasites. Une des conséquences de cet essor a été de délaisser les deux autres options du triptyque décrit.

Toutefois, l'inquiétude croissante des consommateurs sur l'emploi d'intrants chimiques en élevage, la prise en compte nouvelle de possibles impacts écologiques de telles molécules (Lumaret et Errouissi 2002) et surtout, le développement constant, à l'échelle mondiale des résistances aux antiinfectieux de manière générale, et aux antiparasitaires en particulier (Kaplan 2004), a servi de rappel à l'ordre en montrant à quel point un recours quasi exclusif aux traitements fondés sur la chimiothérapie ne représente une solution qu'à très court terme (Waller 2006a). Les solutions répondant aux deux autres principes énoncés ne sont donc pas à négliger pour bâtir une approche intégrée, a priori plus compatible avec le concept de durabilité (Waller et Thamsborg 2004).

Dans ce contexte, il n'est pas anodin de relever que les principes fondateurs de l'Agriculture Biologique (AB) et les recommandations qui en découlent participent de cette synthèse entre les trois principes précédents et privilégient une gestion intégrée des pathologies, prenant en compte le lien au sol, la génétique des animaux, la qualité de l'alimentation et préconisant, seulement en 
dernière intention, une utilisation raisonnée des traitements, lorsque les deux autres options se sont avérées inopérantes (ou inexistantes). Par rapport au REPAB-F, les nouvelles recommandations européennes $\mathrm{CE}$ $n^{\circ} 889 / 2008$, sont moins restrictives sur l'utilisation de traitements allopathiques de synthèse mais restent dans le même esprit. Diverses méthodes ou techniques alternatives aux traitements ont donc été remises à l'ordre du jour et adaptées en fonction des systèmes de production ou des modes de conduite. Il est juste de mentionner que cette tendance existe aussi en élevage conventionnel et que bien des résultats évoqués ici ont été obtenus en conduite hors AB.

La mise en parallèle des principes généraux de gestion des maladies et des recommandations de l'ancien cahier des charges français servira de trame à cet article (Decante et Sans 2001) et nous commenterons les modifications introduites par les nouvelles normes européennes qui s'appliquent dans tous les pays de l'Union. Pour illustrer les aspects d'une telle approche analytique, nous limiterons notre propos à l'élevage des ruminants puisque des articles spécifiques sont consacrés aux productions porcines ou de volailles biologiques dans ce numéro spécial d'Inra Productions Animales consacré à l'Elevage bio. De plus, les exemples retenus se rapporteront aux infestations pas des helminthes, notamment par les Strongles Gastro-Intestinaux (SGI), qui sont régulièrement identifiés comme un risque parasitaire majeur à gérer en élevage $A B$ des ruminants (Lund et Algers 2003, Cabaret 2004). Ce sont les SGI pour qui les données sur les modes de contrôle alternatifs sont les plus abondantes (Thamsborg et al 1999, Waller et Thamsborg 2004).

\section{1 / Gérer le pâturage pour limiter le parasitisme hel- minthique}

Les systèmes d'élevage doivent reposer sur une exploitation maximale des pâturages et l'accès des animaux de rente à des espaces en plein air est favorisé (REPAB 4.7). Le règlement européen $C E n^{\circ} 889 / 2008$ est un peu moins directif sur l'usage des pâturages : dans la majorité des situations, les animaux doivent pouvoir accéder à des aires d'exercice ou de pacage en plein air lorsque les conditions climatiques le permettent, et ces aires de plein air doivent en principe être gérées selon un programme de rotation approprié.

Toutefois, quelle que soit l'espèce, il est recommandé de limiter le nombre d'animaux par unité de surface pour assurer une gestion intégrée des productions (REPAB 1.4 Lien au sol) et la gestion préventive des maladies suppose le maintien de densités animales appropriées (REPAB 5.1, 5.2).

Ces deux recommandations illustrent l'ambivalence intrinsèque associée à l'exploitation de la principale ressource nutritionnelle naturelle des ruminants. Le pâturage constitue la ration de base des herbivores mais il est aussi la source potentielle majeure d'éléments infectieux, notamment de parasites. Dans la maîtrise du parasitisme helminthique lié à l'herbe, cette ambivalence a depuis longtemps été intégrée en préconisant des méthodes raisonnées de gestion du pâturage. De manière liminaire, même si ce point n'est que rarement explicitement mentionné, il faut souligner que ces méthodes ne correspondent qu'à un objectif secondaire, après les impératifs liés à la nutrition des animaux et à la constitution de stocks fourragers pour les périodes de rupture.

L'objectif général de la gestion du pâturage est de minimiser le contact entre les animaux les plus sensibles ou réceptifs aux infestations (les jeunes) et les éléments parasites afin de limiter les infestations à des niveaux compatibles avec les objectifs économiques de l'élevage, sans conséquences majeures sur le bien-être. Un des moyens les plus efficaces pour ce faire est de réduire le chargement par hectare (Saul 1996, Etter et al 2000a), ce qui rejoint la notion de «lien au sol» visant à limiter le nombre d'animaux par unité de surface pour éviter tout problème lié au surpâturage. Par ailleurs, plusieurs méthodes, souvent connues de longue date, permettent aussi un assainissement partiel des parcelles en cherchant à éviter, diluer ou tuer les éléments infestants (Michel 1976, Younie et al 2004). Le succès de ces modes de gestion en situations d'élevage diversifiées suppose une bonne connaissance de la biologie des helminthes.

EVITER : Assainissement par mise au repos des parcelles ; une rotation raisonnée des pâturages réduit-elle le risque parasitaire?

La viabilité et le pouvoir infestant des larves infestantes (L3) de SGI présen- tes dans le milieu extérieur diminuent au cours du temps. De plus, des périodes prolongées de sécheresse ou de gel sont peu propices à la survie des L3 et accélèrent leur mortalité. Une mise au repos des parcelles (sans pâturage) peut donc contribuer à un assainissement relatif. En outre, l'exploitation de retour de fauches est aussi associée à un moindre risque du fait de l'exposition des L3 au soleil. Le retournement par labour des prairies est aussi une mesure envisageable lors de forte contamination du milieu extérieur car elle réduit considérablement la charge en L3 : une prairie retournée tous les 2 à 3 ans permet de maintenir un niveau modéré de parasitisme par les SGI. Cette pratique a aussi des avantages dans la lutte contre les mollusques terrestres, hôtes intermédiaires des petits strongles respiratoires.

La gestion du pâturage comme levier de contrôle des SGI est plus répandu en élevage biologique que conventionnel (Cabaret et al 2009) et certaines stratégies développées par des éleveurs biologiques sont assez efficaces pour contenir le parasitisme par les strongles (Cabaret et al 2002). Dans certains systèmes, des agneaux nés de janvier à mars, sont mis au pâturage avec leurs mères sur des prairies utilisées l'année précédente jusqu'en début d'été. Après sevrage, ils ont alors accès à des parcelles de repousses puis à des cultures annuelles (navets...) puis sont finis en bergerie de septembre à novembre. Entre juillet et septembre, plus de 50\% des agneaux ont atteint leurs poids de vente, le reste étant fini dans la deuxième période.

En régions tempérées, la survie des L3 dans le milieu extérieur est longue, allant de 3 mois à plus de 12 mois selon les espèces de SGI. Ce constat permet une évaluation des temps de repos nécessaires pour aboutir à l'assainissement «naturel» des parcelles : seules des mises au repos prolongées peuvent apporter une réduction notable de la densité parasitaire. En conséquence, de manière générale, des rotations sur des durées courtes se sont révélées peu efficaces pour réduire la pression parasitaire (Hoste et al 1999). De plus, ces mesures sont souvent peu compatibles avec les objectifs agronomiques. On s'oriente désormais vers des modèles $\mathrm{d}$ 'analyse des systèmes de conduite de type HACCP pour intégrer la complexité du risque parasitaire en tenant compte des diversités de situations d'élevage rencontrées (Patout et Devimeux 2005, Le Frileux et al 2006). 
Figure 2. Le pâturage mixte, alterné ou simultané, entre bovins et petits ruminants est une des méthodes de gestion ayant montré une efficacité pour réduire le parasitisme par les strongles gastro-intestinaux.

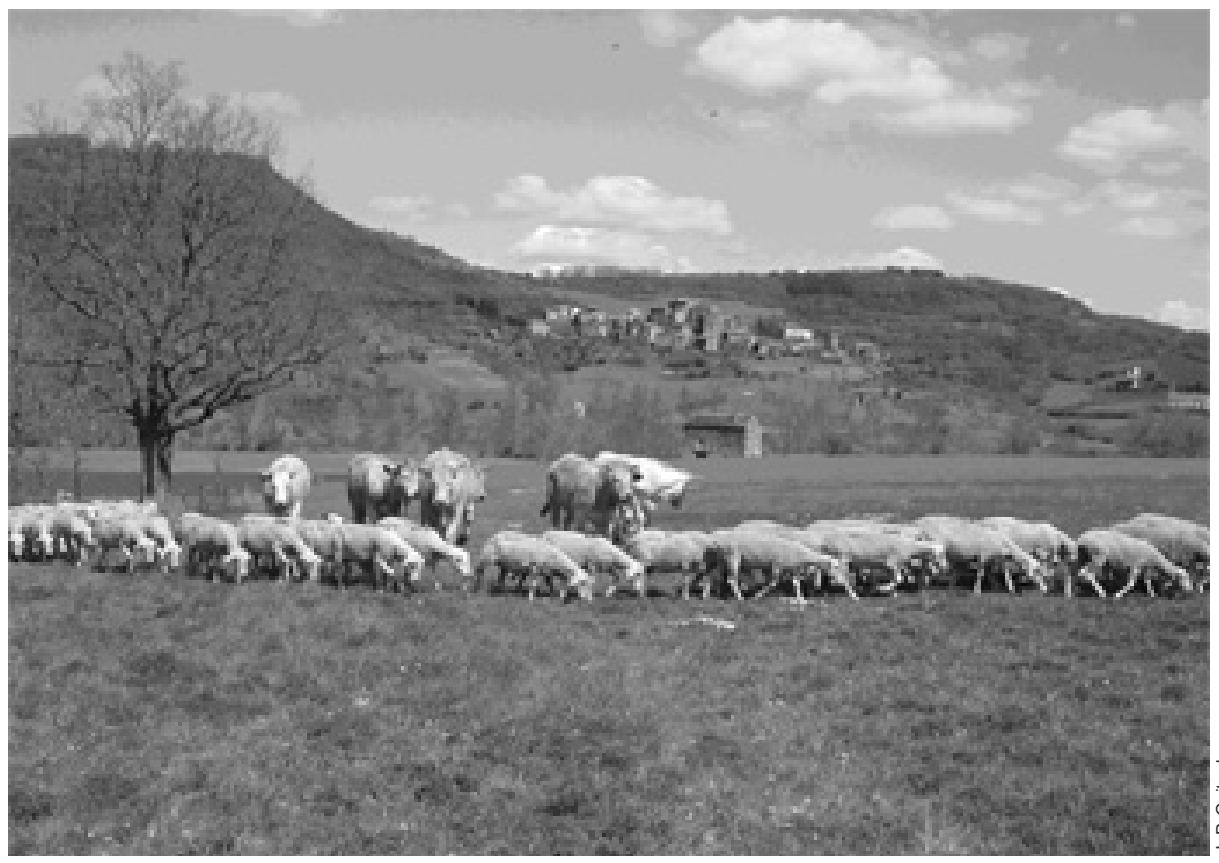

A l'inverse, en zones tropicales, la survie des L3 des principales espèces est beaucoup plus courte. Ainsi, une rotation des parcelles avec retour à intervalle de 30 à 45 jours a donné des résultats beaucoup plus probants (Barger et al 1994, Aumont et al 1997). Cette comparaison illustre comment la connaissance des traits de vie des nématodes est indispensable pour adapter les méthodes de lutte selon les conditions locales (Barger 1999).

DILUER ET TUER : Assainissement par pâturage mixte ou alterné

Les espèces de cestodes et de nématodes des bovins diffèrent de celles des petits ruminants. Cette spécificité relativement étroite est à la base d'une autre méthode de contrôle fondée sur le pâturage mixte entre bovins et petits ruminants [voire entre chevaux et ruminants ou porcs et ruminants (Thamsborg et al 1999)]. L'exploitation alternée ou simultanée des mêmes parcelles par des hôtes différents conduit au «nettoyage» du milieu puisque, en théorie, les formes infestantes d'un parasite ingérées par un hôte inadéquat ne s'installent pas et meurent (figure 2).

Les conséquences du pâturage mixte simultané entre brebis Lacaune et bovins sur le parasitisme des ovins ont été étudiées pendant 3 ans en système de conduite AB, dans le Sud du Massif Central. Cette pratique a été associée de manière répétée, à des excrétions fécales d'œufs de SGI réduites de - $30 \%$ (année 1) à - 60\% (année 2 et 3) (figure 3) ; les baisses étant marquées en fin de saison. De plus, la comparaison des résultats de coprocultures ou des données physiopathologiques (hématocrite) suggèrent que le moindre parasitisme des brebis associées aux bovins concerne surtout un effet spécifique sur Haemonchus contortus, un strongle hématophage très pathogène. A l'inverse, aucun effet sur les infestations des ovins par Oestrus ovis, une myase des voies respiratoires supérieures, n'a été constaté. Chez les bovins, le parasitisme par les strongles est resté faible au cours des 3 ans. Ces résultats corroborent ceux d'études, en zones tempérées (Bairden et al 1995) ou tropicales (Southcott et Barger 1975, Mahieu et al 1997) et confirment l'intérêt de ces pratiques de pâturage commun entre grands et petits ruminants pour maîtriser le parasitisme par des nématodes (Hoste et al 2003). Deux inconvénients potentiels sont toutefois à considérer : 1/ 1'adaptation de SGI à de nouveaux hôtes a déjà été observée (Armour et al 1988) ; 2/ le caractère ubiquiste des trématodes (douves) voire de certains nématodes du genre Trichostrongylus est à garder en mémoire. La méthode parait donc globalement bénéfique mais nécessite une surveillance accentuée vis-à-vis de certains risques spécifiques bien identifiés.
TUER les éléments infestants : un exemple de lutte biologique.

L'application d'amendements chimiques (cyanamide calcique, urée...) sur parcelles est peu efficace pour la destruction massive de L3 de SGI et n'est pas compatible avec les principes de l'AB.

A l'inverse, des résultats concordants ont illustré les potentialités offertes par la lutte biologique, fondée sur l'exploitation des propriétés de diverses espèces de champignons microscopiques nématophages, qui peuvent tuer les larves infestantes de nématodes digestifs dans les fèces (Larsen 2000). En raison de la capacité de ses spores à coloniser les fèces, à germer et à conserver une activité nématophage après passage digestif et de son efficacité vis-à-vis d'un large spectre de strongles digestifs chez les principales espèces de rente, l'espèce, Dunddigtonia flagrans a fait l'objet de la plupart des études récentes. Son application en conditions d'élevage suppose l'apport quotidien de spores, pendant 6 à 8 semaines, en début de saison d'herbe, pour "geler» le développement des L3 et freiner la dynamique des infestations. La plupart des données actuelles sur l'efficacité, l'absence de nocivité apparente pour l'animal ou l'environnement (Paraud et al 2007) sont favorables, indiquant des réductions d'infestivité des fèces atteignant 90\% (Larsen 2000, Paraud et al 2005). Cependant, l'écueil technologique visant à faciliter la distribution individuelle des spores sur une période prolongée reste à lever avant d'envisager une exploitation commerciale de cette méthode.

\section{2 / Prévenir en améliorant la réponse de l'hôte}

En théorie, trois moyens principaux sont à disposition pour favoriser la capacité des animaux à mieux affronter les parasites (résistance) ou à moins pâtir de leur présence (résilience) : i) vacciner (prophylaxie médicale) ; ii) sélectionner, sur des bases génétiques, les races ou lignées montrant une meilleure réponse au parasitisme ; iii) améliorer la nutrition.

\section{1 / Vaccination}

En dépit d'un premier succès obtenu avec un vaccin vivant atténué contre la dictyocaulose bovine, le développement de vaccins contre les helminthes 
Figure 3. Excrétion fécale comparée des brebis Lacaune élevée à l'herbe avec ou sans bovins au cours de trois années de pâturage. Histogramme blanc : brebis avec des bovins ; histogramme rouge : brebis seules.

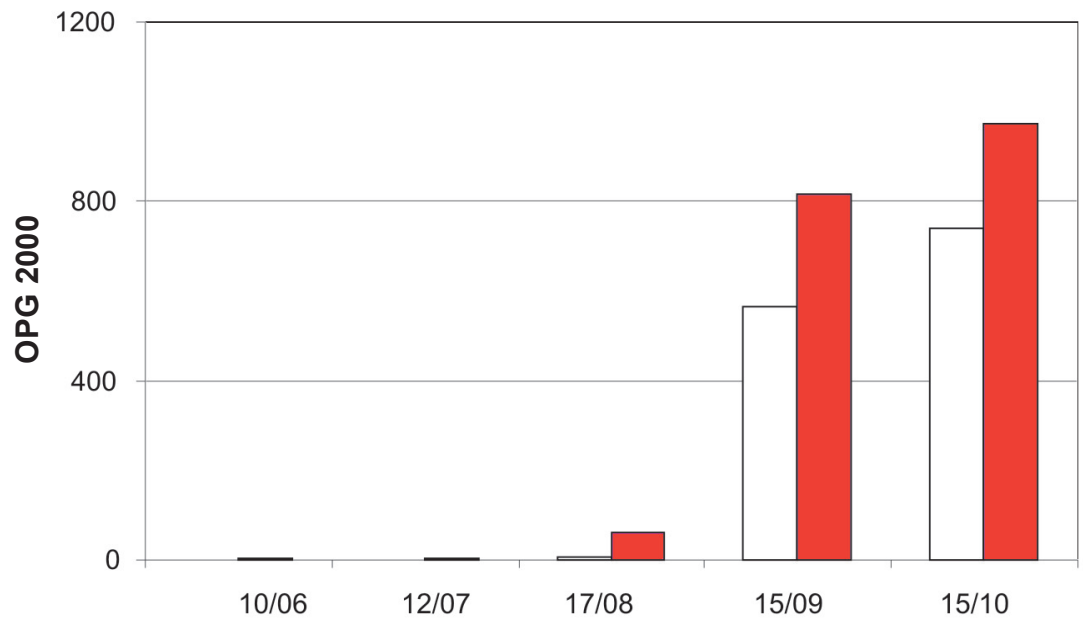

Date de prélèvements

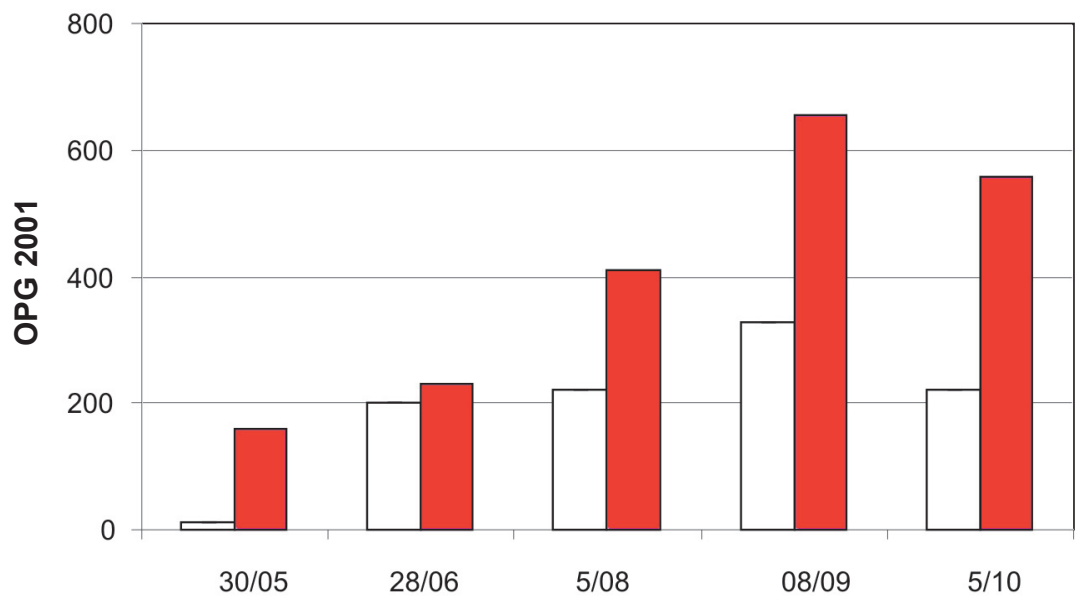

Date de prélèvements

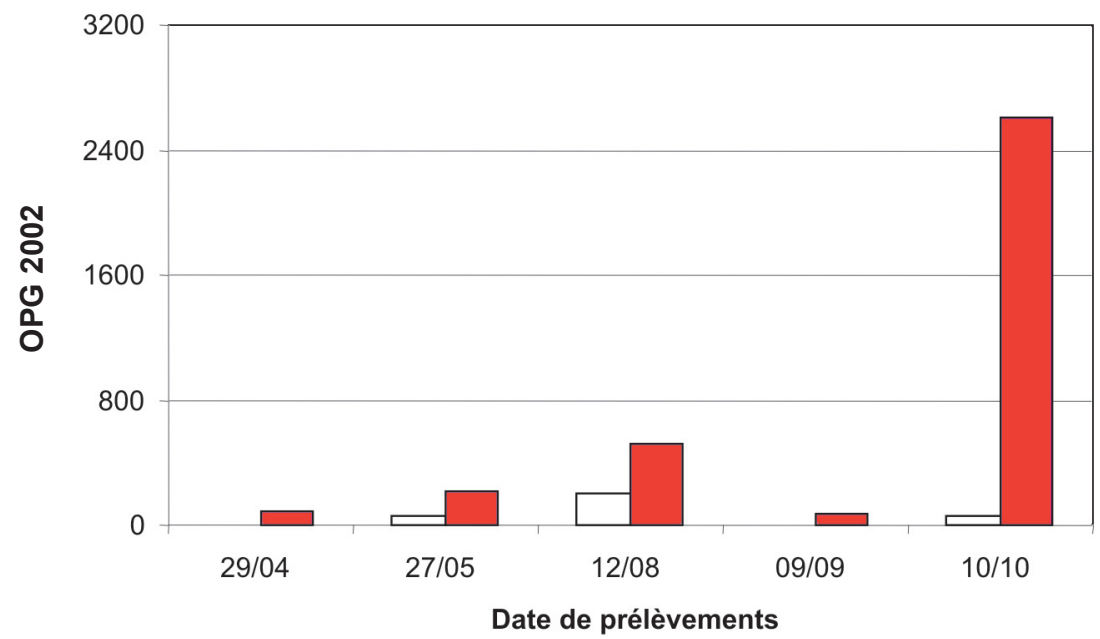

des ruminants demeure du domaine de la recherche (Smith et Zarlenga 2006). Pour les SGI, les avancées principales ont été obtenues vis-à-vis d'H. contortus, en raison de son importance économique. Les travaux menés ont permis à conceptuels subsistent qui en limitent les applications. Actuellement, aucun vaccin commercial n'existe.

\section{2 / Génétique}

De manière générale, l'existence d'une composante génétique dans la variabilité de réponse aux nématodes a été établie chez les deux espèces de petits ruminants soit entre races ovines ou caprines, soit entre individus d'une même race (lignées) (Woolaston et Baker 1996, Gruner et al 1998, Vagenas et al 2002). La plupart des études ont porté sur la résistance des animaux (leur aptitude à limiter les populations de vers ou à perturber leur biologie). Les données comparées sur la résilience (aptitude à supporter les effets négatifs du parasitisme) entre races ou individus sont moins nombreuses (Bisset et al 1996). Enfin, les informations comparatives collectées sur les races locales, autochtones, restent trop rares.

L'excrétion fécale des œufs de nématodes a été le critère le plus utilisé pour comparer la résistance des individus (Douch et al 1996, Gruner et al 1998). Cependant, d'autres marqueurs phénotypiques (paramètres pathophysiologiques (hématocrite) ou immunologiques (taux d'anticorps)) ont parfois été proposés pour opérer la sélection (Douch et al 1996). Les études récentes s'orientent plutôt vers la recherche de marqueurs moléculaires de la résistance (Douch et al 1996, Bishop et Morris 2007).

Ces sélections d'animaux résistants constituent des programmes inscrits sur le long terme, qui doivent prendre en compte les conditions locales d'élevage, la disponibilité des races présentes et les objectifs de l'élevage (Simm et al 1996, Pomroy 2006). De plus, cette sélection d'animaux résistants aux SGI peut également présenter des limites telles que le risque d'une augmentation de sensibilité des hôtes à d'autres pathogènes que les strongles (Gruner et al 1998) ou un effet défavorable sur la productivité (Mc Ewan et al 1992, Stear et Murray 1994). En Australie et Nouvelle-Zélande, cette option s'est d'ores et déjà traduite par des schémas de sélection appliqués aux principales races ovines. En Europe, la diversité des races locales ou régionales rend cette entreprise multiple et donc coûteuse. Enfin, avec le temps, les nématodes seraient susceptibles de contourner ces résistances, comme cela a été montré sur les parasites de plantes. 


\section{3 / Nutrition}

\section{a) Ajustement adaptation quantita- tive}

L'importance des interactions entre nutrition et parasitisme chez les ruminants a été identifiée très tôt (Clunies Ross et Gordon 1933). L'idée a reçu depuis de multiples confirmations, notamment lors d'infestations par les SGI chez les ovins ou les caprins (Coop et Kyriazakis 1999, Etter et al 2000b, Steel 2003). L'essentiel des résultats ont porté sur la composante protéique de la ration, en montrant comment des complémentations adaptées permettaient de limiter le parasitisme et ses effets néfastes. Cette focalisation sur les protéines s'explique d'abord par le fait que le métabolisme azoté est le premier perturbé lors de parasitisme gastro-intestinal (Bown et al 1991). De plus, en zone tempérée, les protéines sont souvent le principal facteur nutritionnel limitant chez les ruminants, ce qui, selon le modèle théorique de Coop et Kyriazakis (1999), en fait une cible de choix pour les ajustements de complémentations. Les bénéfices potentiels associés à la manipulation de la composante énergétique de la ration ont été beaucoup moins explorés (Torres Acosta et al 2004, 2006). Enfin, les interactions entre les apports alimentaires de macro ou d'oligoéléments ou de vitamines et les composants de la réponse immune de l'hôte ont été l'objet de multiples études théoriques sur modèles murins sans beaucoup de confirmations chez les ruminants.

Malgré l'accumulation de données fondamentales, la connaissance de ces interactions reste par bien des aspects insuffisante pour exploiter de façon efficace et économe ce levier en élevage dans le cas du parasitisme helminthique des ruminants. Plusieurs raisons sont à incriminer. Pour ne prendre que le seul exemple des protéines de la ration, le manque d'indicateurs individuels précis pour mesurer les déficits provoqués par les vers rend difficile des ajustements précis. De plus, en $\mathrm{AB}$, l'accès à des ressources protéiques suffisantes et leurs coûts éventuels constituent un autre facteur limitant à cette méthode sauf en systèmes fourragers comportant beaucoup de légumineuses.

\section{b) Notion d'alicaments/nutricaments}

Les alicaments/nutricaments sont des plantes contenant des métabolites secondaires dont 1'exploitation se justifie plus en raison de leurs propriétés sur la santé des animaux que pour leur valeur nutritionnelle.

Depuis 10 ans, un certain nombre de plantes bioactives ont été identifiées dont l'activité anthelminthique a été établie de manière répétée (Hoste et al 2006). A l'exception notable de la chicorée (Cichoryum intybus ; Composées) (Marley et al 2003), ces plantes appartiennent à la famille des Légumineuses (sulla, lotier pédonculé (Lotus pedunculatus) ou corniculé (L. corniculatus), sainfoin (Onobrychis viciifoliae), sericea (Lespedeza sericea)). Toutefois l'activité anthelminthique de plantes composant le couvert pastoral méditerranéen commence à être explorée (Paolini et al 2004, Osoro et al 2007). Dans le cas des plantes fourragères, les propriétés anthelminthiques ont été associées à la présence de certains composés biochimiques spécifiques : sesquiterpènes lactoniques pour la chicorée ou tannins condensés pour les légumineuses fourragères (Hoste et al 2006). Ces composés polyphénoliques ont fait l'objet des recherches les plus poussées pour démontrer leur efficacité ou en comprendre les modes d'action.

De multiples études in vitro ou in vivo, chez les ovins ou les caprins, ont objectivé l'activité des tannins sur les principaux nématodes de l'abomasum ou de l'intestin (Niezen et al 1996, Athanasiadou et al 2000, 2001, Shaik et al 2006). Chez l'animal, la consommation de ces fourrages a été associée à deux impacts principaux sur la biologie des vers : 1/ un effet sur les larves infestantes en réduisant leur installation chez l'hôte ; 2/ un effet sur les vers adultes affectant surtout leur reproduction et l'excrétion des œufs, donc contribuant à réduire la contamination du milieu extérieur (Hoste et al 2006). A l'inverse des anthelminthiques chimiques, l'efficacité des tannins n'est jamais absolue, les baisses maximales d'excrétion d'œufs signalées atteignant 60 à $70 \%$ (Heckendorn et al 2006, Lange et al 2006, Shaik et al 2006). De plus, ces effets sur les populations de vers ont souvent été associés à une meilleure résilience de l'hôte au parasitisme, s'exprimant par des perturbations physiopathologiques ou des pertes de production réduites (Niezen et al 1996, Paolini et al 2005).

Toutefois, avant d'envisager une application pertinente en élevage de ces plantes bioactives, plusieurs questions restent à résoudre. Il faut notamment comprendre quels facteurs liés à la plante, à l'hôte ou aux parasites (espèces, stade) expliquent la variabilité des résultats observés. Les concentrations minimales de tannins requises et les différences potentielles d'activité selon la nature des tannins en cause restent largement à explorer. Enfin, malgré certaines avancées récentes (Brunet et al 2007), les mécanismes d'action sur les vers restent mal élucidés.

\section{3 / Traiter autrement}

Les produits de phytothérapie, d'aromathérapie ou d'homéopathie ainsi que les oligoélements sont à utiliser de préférence aux médicaments vétérinaires allopathiques chimiques de synthèse à condition d'avoir un effet thérapeutique réel sur l'espèce animale concernée et aux fins spécifiques de traitements (REPAB 5.4) Le règlement européen reprend les mêmes propositions.

De nombreux traitements contre les strongles gastro-intestinaux, relevant de ces alternatives à l'allopathie sont répertoriés. Toutefois, les études visant à démontrer leur efficacité sur des bases objectives restent rares. Elles se heurtent souvent à des difficultés méthodologiques pour évaluer les effets. La variabilité des ressources exploitées et des produits proposés doit également être reconnue. Enfin, la connaissance des mécanismes d'action impliqués est généralement absente.

\section{1 / Homéopathie}

L'homéopathie repose sur le traitement individualisé à très faible dose de drogue, en relation avec l'ensemble des symptômes présents. Cela est difficile en médecine vétérinaire puisque des traitements de groupes sont généralement la règle. Toutefois, dans la mesure où les animaux seraient traités à tort, l'absence d'action est attendue et les problèmes de résidus nuls : il n'y a donc pas de conséquences néfastes. Les principaux traitements répertoriés contre les strongles sont Cina (extrait d'Artemisia cina, armoise de Chine, Cabaret 1996) et Teucrium (extrait de Teucrium marum, germandrée maritime, Gibbons 2002). La difficulté résulte dans l'évaluation des effets. Ainsi dans le cas où des lots traités et non traités sont constitués a priori, il n'est pas sûr que tous les individus du lot traité présentent les symptômes justifiant leur inclusion dans ce groupe aux yeux d'un thérapeute homéopathe. Tabel et al (2008) proposent une 
méthodologie qui repose sur la constitution de lots a posteriori selon des règles strictes préétablies. Les lots sont constitués sur des critères de santé (niveau d'anémie et de diarrhée, capacité à la croissance chez des agneaux) selon une méthodologie statistique et l'efficacité parasitaire peut alors être estimée. Un énorme travail de réflexion méthodologique reste donc à mener pour aboutir à une évaluation objective des traitements homéopathiques destinés à limiter l'effet des SGI sur la santé des animaux, qui soit acceptable à la fois par les expérimentateurs analytiques et les homéopathes.

\section{2 / Aromathérapie}

Utiliser des solutions alternatives pour éliminer des parasites intestinaux se limite le plus souvent au seul choix de produits naturels dont on espère qu'ils seront sans toxicité et sans induction de phénomènes de résistance. A cet égard les huiles essentielles sont particulièrement représentatives de cette méprise. Pour mieux comprendre la nature exacte de leurs usages, il convient de procéder à quelques rappels.

a) Composition des huiles essentielles réputées pour leurs propriétés antiparasitaires

Toutes les Huiles Essentielles (HE) sont complexes, composées d'une dizaine de molécules dominantes accompagnées d'une cinquantaine d'autres plus secondaires. Les huiles essentielles les plus réputées pour leur activité vermicide contiennent des phénols, des alcools, des cétones, des esters ou des terpènes.

In vitro, ces composants agissent soit par action directe sur la cuticule des parasites (phénols, alcools) soit par effet neurotoxique (cétones) (Franchome et Penoel 1990), soit par des processus plus complexes en affectant la nutrition ou la reproduction des parasites adultes (Regnault-Roger 2002). Il est possible de mesurer, in vitro, la concentration minimale d'huiles essentielles nécessaires pour tuer les parasites mais cela a surtout été le cas pour les parasites unicellulaires (Grosmond 1999). Ces investigations ont été largement pratiquées sur Histomonas meleagridis et les diverses coccidies lorsque la législation européenne sur les additifs alimentaires a été modifiée en 2004.

\section{b) Expérience chez les animaux}

Chez les animaux de rente, il est souvent difficile d'établir une relation claire entre le niveau d'infestation parasitaire, l'indice de consommation et la production. La mesure des effets favorables des huiles essentielles peut donc se faire sur la base de critères soit parasitologiques, soit zootechniques.

L'activité vermicide effective des huiles essentielles se heurte aujourd'hui à leur mauvaise tolérance digestive aux doses où elles sont efficaces et à la difficulté à les véhiculer jusqu'au siège des infestations parasitaires du fait de leur très forte solubilité dans les phospholipides de la paroi digestive. Actuellement, nous n'avons constaté d'activité vermicide réelle que sur les strongles gastro-intestinaux des petits ruminants ou par la très forte réduction de ponte du paramphistome chez les bovins (Mage et Grosmond 2009). Dans les deux cas, il s'agit d'une progression courte des huiles essentielles dans le tube digestif avec mise en contact très rapide avec les parasites et l'effet n'est constaté qu'après administration répétée sur deux ou trois jours. Il n'existe pas d'activité vermicide mesurée avec une seule administration d'huiles essentielles sauf à être très proche des doses toxiques ou agressives pour la muqueuse digestive.

Il en est tout autrement lorsque l'effet antiparasitaire recherché est mesuré sur les performances zootechniques. On constate en effet, surtout lors d'infestations coccidiennes, une réelle différence de performances entre lots traités et témoins (Grosmond et Hezouan 2009), sans qu'existe de corrélation entre le nombre d'ookystes émis et les gains de croissance. Le bénéfice associé à l'usage des huiles essentielles semble donc à chercher ailleurs que dans une activité antiparasitaire stricto sensu.

c) Comment concevoir la place des huiles essentielles parmi les solutions alternatives de gestion de la santé animale?

En raison des interactions qu'elle peut susciter, il est fondamental de prendre en compte la complexité de composition des huiles essentielles et ne jamais se limiter à la molécule dominante de chaque HE. Cette complexité s'avère utile car elle exerce des effets régulateurs sur le système nerveux autonome, le système endocrinien (Jain et Sachdev 1995), le système immunitaire (Lamm et Riggs 2000) ou les grandes fonctions : digestive (Debelmas et Rochat 1967), hépatique (Youdim et Deans 2000), etc.

Vouloir réduire l'activité d'une huile essentielle à un effet de type allopathique nécessite des concentrations élevées et dangereuses de principes actifs. L'usage d'《analogues naturels», composés de synthèse reproduisant la molécule principale d'une huile essentielle, à des fins antiparasitaires montre aujourd'hui ses limites en particulier vis-à-vis d'autres molécules environnementales comme les pesticides (Ellis et al 1997). Les mélanges d'huiles essentielles à des concentrations modérées et bien tolérées, peuvent apparaître comme des régulateurs des grandes fonctions et comme des auxiliaires précieux dans la gestion de la santé animale en complément de nouvelles techniques d'élevage. En règle générale, les HE ne sont pas, à ce jour, considérées comme des médicaments, ce qui rend bien compte de leur rôle fondamental

La consommation de légumineuses riches en tannins, comme le sainfoin, est une des alternatives actuelles explorées pour compléter l'action des anthelminthiques chimiques.

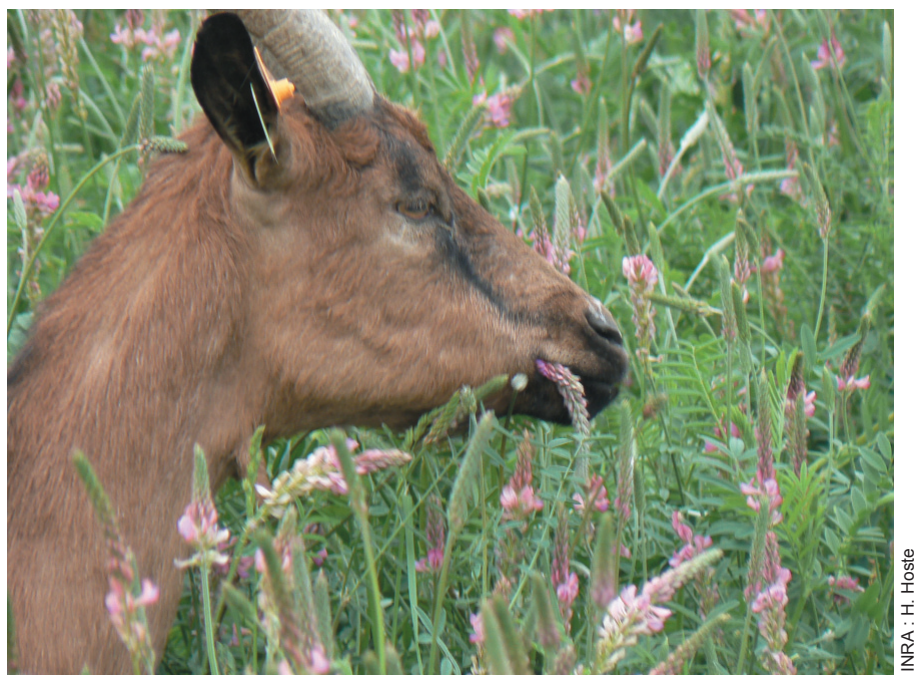


Figure 4. En $A B$, les traitements antiparasitaires ne doivent plus constituer la pierre angulaire de la maitrise du parasitisme qui doit plutôt reposer sur des mesures visant à réduire les contacts avec les éléments infestants ou à améliorer la réponse de l'hôte. Restreindre l'usage des molécules chimiques demeure un objectif valide en élevage $A B$.

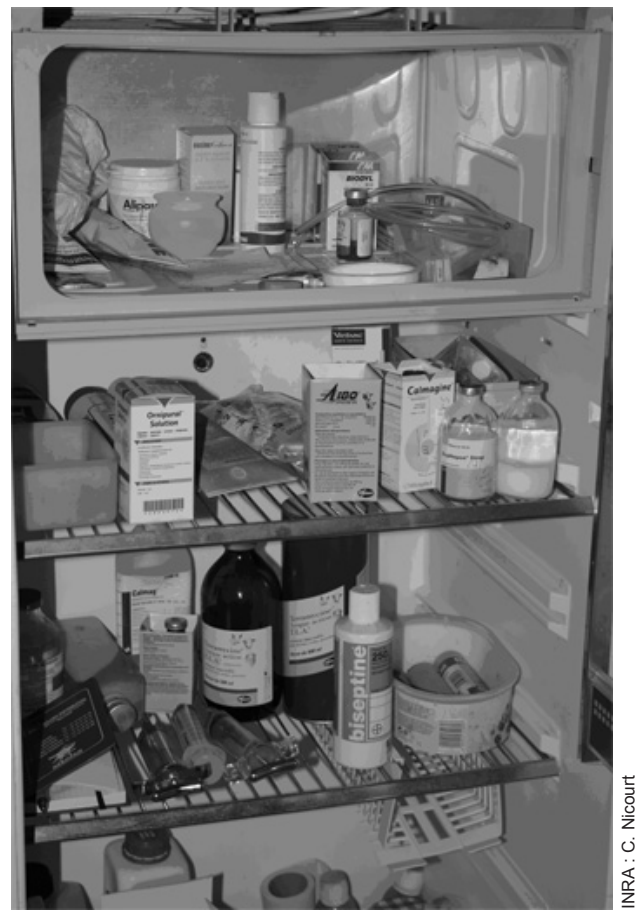

comme moyens de régulation des grandes fonctions.

\section{3 / Phytothérapie}

Les ressources phytothérapiques sont tirées des savoirs populaires et ont été parfois formalisées dans les pratiques de la médecine vétérinaire ancienne (Cerbelaud 1922). Ainsi, les remèdes végétaux suivants étaient répertoriés par Cerbelaud chez l'animal ou Leclerc (1922) chez l'Homme, certains étant encore à la base de bien des formulations actuelles : rhizome de fougère mâle, kamala, noix d'arec, écorce de racines de grenadier, semences de courge, chénopode, semencontra, absinthe, tanaisie. L'introduction de l'usage des huiles essentielles est postérieure.

L'efficacité des produits de la phytothérapie doit être évaluée de manière usuelle, comme pour les anthelminthiques de synthèse par exemple. Les validations à dire d'éleveurs sont à considérer mais ne sont pas des preuves d'efficacité. En effet, l'évaluation des produits de phytothérapie par les éleveurs est délicate, car il leur est difficile de mesurer les niveaux d'infestation des troupeaux sans recours au laboratoire. Pour prendre un exemple, un traitement commercial alterna- tif utilisé contre le cestode des agneaux Moniezia expansa est très apprécié des éleveurs. Toutefois, deux évaluations en conditions contrôlées au pâturage n'ont pas mis en évidence de différences d'efficacité entre lots traités et non traités (Cabaret et al 2005). Cette contradiction apparente peut être levée. L'infestation des agneaux par Moniezia est particulièrement importante en fin de printemps puis, s'installe peu à peu une forte protection contre ce parasite qui conduit à une quasi-disparition des infestations deux mois après leur apparition. Dans ces conditions, tout traitement ou absence de traitement se confond sur le moyen terme. Par contre, le produit provoque une plus grande excrétion fécale des anneaux du Taenidé, dans les jours qui suivent l'administration. Cette activité très partielle mais visible amène l'éleveur à croire à l'efficacité du produit. La conjonction des deux phénomènes (rémission naturelle de la monieziose et excrétion visible $\mathrm{du}$ parasite) conduit à une bonne acceptation du produit sans qu'une efficacité réelle soit démontrée. De manière générale, l'évaluation de divers produits fondée sur des mesures parasitaires objectives ont souvent donné des résultats décevants (Bouilhol et al 2003)

\section{4 / Nouvelles modalités d'utili- sation des anthelminthiques de synthèse en $A B$}

\section{- Pas d'emploi des traitements chi- miques de synthèse de manière pré- ventive}

Cette règle vaut pour l'ensemble des parasites. Toutefois, il est connu que les brebis en fin de gestation et début de lactation excrètent un nombre plus élevé d'œufs de SGI dans les fèces ce qui aboutit à une infestation très supérieure des agneaux (Athanasiadou et al 2007). Le cahier des charges est donc un frein à la pratique consistant à traiter avant la mise à l'herbe des seules brebis ayant mis bas.

\section{- Restriction du nombre de traite- ments en fonction des espèces en cause}

Dans le REPAB-F, le nombre de traitements anthelminthiques de synthèse devait être inférieur ou égal à trois pour les agneaux. Les nouvelles recommandations européennes CE $n^{\circ}$ 889/2008 sont beaucoup plus permissives à cet égard: «En dehors des vaccinations, des traitements antiparasitaires et des plans d'éradication obligatoires, si un animal ou un groupe d'animaux reçoit au cours d'une période de douze mois plus de trois traitements à base de médicaments vétérinaires allopathiques chimiques de synthèse ou d'antibiotiques, ou plus d'un traitement si leur cycle de vie productive est inférieur à un an, les animaux concernés ou les produits obtenus à partir de ces animaux ne peuvent être vendus en tant que produits biologiques». Cela revient à ne pas imposer de limites aux traitements antiparasitaires. Il est difficile de comprendre cette position, sachant que des normes plus strictes peuvent être appliquées sans difficultés majeures chez les ruminants.

Quoiqu'il en soit, l'emploi modéré des traitements doit rester la règle pour éviter le développement de résistances chez les vers et répondre aux attentes des consommateurs (figure 4). Une telle restriction reste tout à fait praticable : dans une conversion $\mathrm{AB}$ en moyenne montagne les animaux exclus du circuit $A B$ pour cause d'excès de traitements ont été très rares $(<2 \%)$ (Cabaret 2004). De même, une enquête réalisée en fermes ovines $\mathrm{AB}$ ou conventionnelle en Auvergne/Limousin indique que les agneaux en production viande AB sont traités par des anthelminthiques de synthèse 1 à 2 fois au cours de leur carrière, contrairement aux 7 à 8 fois rencontrées chez les fermiers conventionnels (Cabaret et al 2009). 
Figure 5. La coproscopie à partir des fèces d'animaux infestés demeure la méthode de diagnostic de référence pour les strongyloses gastro-intestinales mais d'autres approches fondées sur l'évolution de signes cliniques ou de critères zootechniques sont en cours de validation.

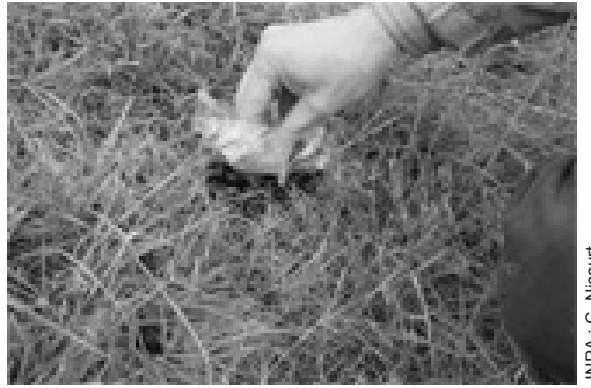

- Ciblage de l'utilisation des anthelminthiques vers les animaux les plus affectés

Cette stratégie (TST : Targeted selective treatment) consiste à appliquer un traitement sélectif sur seulement une partie du troupeau, en ciblant les animaux les plus infestés. L'utilisation des TST est peu probable en élevage conventionnel, demandeur de règles fixes et simples de traitements, alors que les éleveurs ovins $\mathrm{AB}$ sont plus intéressés (Cabaret et al 2009). La TST a fait l'objet de travaux récents en particulier dans un projet européen (Parasite solutions : Parasol) sur les ruminants. La principale difficulté est de détecter les animaux à traiter. Ce repérage peut se fonder sur des méthodes de laboratoire, l'évaluation graduée de signes cliniques ou encore sur l'analyse de données épidémiologiques.

Les coproscopies (figure 5) dans les matières fécales sont un moyen classique mais qui ne peut s'appliquer à des troupeaux importants. L'objectif est donc de substituer des indices facile- ment utilisables par l'éleveur luimême. L'indice d'anémie (FamachaC chez les petits ruminants) est l' exemple de tels indicateurs qui a été le plus utilisé (Van Wyk et Bath 2002) en particulier pour contrôler les infestations par le strongle hématophage, Haemonchus contortus. Des indices de diarrhées ont aussi été proposés (dag-score, salissure de l'arrière train visible facilement dans le cas de diarrhées répétitives ou l'indice de diarrhée instantanée ou Disco, Cabaret 2004) mais leur utilisation pour des traitements individualisés se heurte à la lourdeur des opérations, surtout si plusieurs indicateurs sont nécessaires en raison de faunes helminthiques diversifiées. En revanche, des suivis réalisés sur quelques animaux sentinelles (animaux du troupeau suivis régulièrement au plan parasitologique) permettent de repérer les périodes à risque pour les SGI. Un indice intégrateur, le gain de poids des agneaux en élevage allaitant est un indicateur intéressant mais la charge de travail est importante avec les instruments actuels. Les progrès en matière de mesure électronique des poids et de sélection des animaux selon leurs performances, la mobilité des installations pourrait dans le futur favoriser son usage. Enfin, le repérage de catégories d'animaux à risque (jeunes primipares ou animaux à forte production laitière chez les caprins) (Hoste et al 2002) est également une possibilité qui a été explorée pour réduire l'emploi des anthelminthiques de synthèse.

\section{Conclusions}

Cet exemple sur le développement d'alternatives de lutte contre les strongles gastro-intestinaux illustre plusieurs aspects d'une démarche analytique dans le cadre de l'Agriculture Biologique. Un des principaux ensei- gnements à tirer du développement rapide (Waller 2006a) et de l'expansion des résistances aux molécules anthelminthiques est probablement de valider le bien-fondé d'une approche plus complexe qui correspond aux principes de l'AB déclinés par le règlement communautaire. Même en système conventionnel, la nécessité d'une approche intégrée, combinant plusieurs solutions, si possible relevant des trois principes de maitrise des pathologies, est aujourd'hui largement reconnue et préconisée (Waller 2006b).

Toutefois, cette revue des solutions disponibles ou explorées souligne aussi que des déficits de connaissance ou de méthodologies persistent qui limitent l'apport de réponses adaptées au mode de conduite AB. Il apparait clairement par exemple que les outils méthodologiques employés pour attester de l'efficacité de produits issus de l'homéopathie ou de l'aromathérapie sont mal adaptés. Par ailleurs, les connaissances fondamentales pour appliquer de manière pertinente ces méthodes en élevage restent souvent, au mieux, parcellaires. Enfin, les instruments pour évaluer de manière synthétique et ajuster les démarches combinant ces solutions aux situations de «terrain» restent rares et complexes (Le Frileux et al 2006). Les besoins de recherche/développement pour gérer le parasitisme et, de manière plus générale, les questions sanitaires en $\mathrm{AB}$ sont donc toujours d'actualité, en gardant à l'esprit que leurs répercussions peuvent aussi être d'intérêt pour l'élevage conventionnel.

\section{Remerciements}

Les auteurs remercient le projet européen Marie Curie Healthy Hay pour le soutien financier accordé.

\section{Références}

Armour J., Bairden K, Dalgliesh R, Ibarra Silva A.M., Salman S.K., 1988. Clinical nematodiriasis in calves due to Nematodirus battus. Vet. Rec., 123, 230-231.

Athanasiadou S., Kyriazakis I., Jackson F., Coop R.L., 2000. Effects of short-term exposure to condensed tannins on adult Trichostrongylus colubriformis. Vet. Rec., 146, 728-732.

Athanasiadou S., Kyriazakis I., Jackson F., Coop R.L., 2001. Direct anthelmintic effects of condensed tannins towards different gastrointestinal nematodes of sheep: in vitro and in vivo studies. Vet. Parasitol., 99, 205-219.

Athanasiadou S., Gray D., Younie D., Tzamaloukas O., Jackson F., Kyriazakis I., 2007.
The use of chicory for parasite control in organic ewes and their lambs. Parasitology, 134, 299-307.

Aumont G., Pouillot R., Simon R., Hostache G., Varo H., Barré N., 1997. Parasitisme digestif des petits ruminants dans les Antilles Françaises. INRA Prod. Anim., 10, 79-89.

Bairden K., Armour J., Duncan J., 1995. A 4year study on the effectiveness of alternate grazing of cattle and sheep in the control of bovine parasitic nematodes. Vet. Parasitol., 60, 119-132.

Barger I.A., 1999. The role of epidemiological knowledge and grazing management for helminth control in small ruminants. Int. J. Parasitol., 29, 41-47.
Barger I.A., Siale K., Banks D.J.D., Le Jambre L.F., 1994. Rotational grazing for control of gastrointestinal nematodes of goats in wet tropical environment. Vet. Parasitol., 53, 109-116.

Bishop S.C., Morris C.A., 2007. Genetics of disease resistance in sheep and goats. Small Rum. Res., 70, 48-59.

Bisset S.A., Morris C.A, Squire D.R., Hickey S.M., 1996. Genetics of resilience to nematode parasites in young Romney sheep, use of weight gain under challenge to assess individual anthelmintic treatments. N. Z. J. Agric. Res., 39, 314-323

Bouilhol M., Mage C., Archimbaud E., 2003 Activité antiparasitaire de produits phytothérapiques. CR 2013206. Institut de l'Elevage, 3-4. 
Bown M.D., Poppi D.P., Sykes A.R., 1991. The effect of post-ruminal infusion of protein or energy on the pathophysiology of Trichostrongylus colubriformis infection and body composition in lambs. Aust. J. Agric., 42, 253-267.

Brunet S., Aufrere J., El Babili F., Fouraste I., Hoste H., 2007. The kinetics of exsheathment of infective nematode larvae is disturbed in presence of tannin-rich plant (sainfoin) both in vitro and in vivo. Parasitology, 134, 1253-1262.

Cabaret J., 1996. The homeopathic Cina does not reduce the egg output of digestive-tract nematodes in lambs. Rev. Méd. Vét., 147, 445-446.

Cabaret J., 2004. Parasitisme helminthique en élevage biologique ovin : réalités et moyens de contrôle. INRA Prod. Anim., 17, 145-154.

Cabaret J., Bouilhol M., Mage C., 2002. Managing helminths of ruminants in organic farming. Vet. Res., 33, 625-640.

Cabaret J., Gonnord V., Cortet J., Sauvé C., Ballet J., Tournadre H., 2005. Moniezia chez l'agneaux d'herbe : épidémiologie et tentative de contrôle par un traitement alternatif. Alter Agri, $72,4-7$

Cabaret J., Benoit M., Laignel G., Nicourt C., 2009. Situation and management of internal parasites for conventional and organic meat sheep farmers in Central France: how does it fit for targeted selective treatments? Vet. Parasitol., sous presse.

CC-REPAB-F, 2004. Ministère de l'Agriculture de l'Alimentation de la Pêche et des Affaires Rurales, 74p. http://www.agriculture.gouv.fr/ spip/IMG/pdf/ccrepabfconso a1a2a3a4.pdf

Cerbelaud R., 1922. Manuel vétérinaire ou formulaire des médications rationnelles et des remèdes secrets. Imprimerie Piget et fils, Orléans, France, 1888p.

Clunies Ross I., Gordon H.M., 1933. Nutritional factors affecting the resistance to haemonchosis. Aust. Vet. J., 9, 100-107.

Coop R.L., Kyriazakis I., 1999. Nutritionparasite interaction. Vet. Parasitol., 84, 187-204.

Debelmas AM., Rochat J., 1967. Etude pharmacologique des huiles essentielles, activité antispasmodique. Plantes Médicinales et Phytothérapie, 1, 23-27.

Decante F., Sans P., 2001. Le règlement européen des productions animales biologiques: mode d'emploi du REPAB. Bull. GTV, Numéro Spécial Agriculture Biologique, 36-46.

Douch P.G., Green R.S., Morris C.A., McEwan J.C., Windon R.G., 1996. Phenotypic markers for selection of nematode-resistant sheep. Int. J. Parasitol., 26, 899-911.

Ellis M.D., Siegfried B.D., Spawn B., 1997. The effect of Apistan on honey bee (Apis melli fera L). Reponses to methyl parathion, carbaryl and bifenthrin exposure. Congrès, Arbeitsgemeinschaft der Institute für Bienenforschung e V. Jahrestagung, 44, Jena, Allemagne, 28, 3-4, 123-127, 151-233.

Etter E., Chartier C., Hoste H., Pors I., Lefrileux, Y., Broqua C., Vallade S., Goudeau C., 2000a. Parasitisme par les nématodes du tube digestif et utilisation du pâturage : épidémiologie de l'infestation dans les troupeaux caprins laitiers en France. Epidémiol. Santé Anim., 37, 75-86.

Etter E., Hoste H., Chartier C., Pors I., Koch C., Broqua C., Coutineau H., 2000b. The effect of 2 levels of dietary protein on resistance and resilience of dairy goats experimentally infected with Trichostrongylus colubriformis: comparison between high and low producers. Vet. Res., $31,247-258$

Franchome P., Penoel D., 1990. L'aromathérapie exactement. Jollois édit. Marseille, France, 490p.

Gibbons J., 2002. Alternative methods of internal parasite control in sheep. University of Aberdeen. www.abdn.ac.uk/organic/ organic 34

Grosmond G., 1999. Test in vitro de l'efficacité de 10 H.E. de plantes sur Tetratrichomonas gallinarum. Congrès Mondial Vetérinaire. Lyon, France, 2p.

Grosmond G., Heouzan J., 2009. Gestion de la coccidiose chez l'agneau de bergerie à l'aide d'huiles essentielles. Sem. Vét., Sous presse.

Gruner L., Bouix J., Vu Tien Khang J., 1998. La résistance génétique aux parasitoses internes : exemples de travaux engagés en France et en Pologne. Point Vét., 29, 1129-1137.

Heckendorn F., Häring D.A., Maurer V., Zinsstag J., Langhans W., Hertzberg H., 2006. Effect of sainfoin (Onobrychis viciifolia) silage and hay on established populations of Haemonchus contortus and Cooperia curticei in lambs. Vet. Parasitol., 142, 293-300.

Hoste H., Le Frileux Y., Pommaret A., Gruner, L., Van Quackebeke E., Koch C., 1999. Importance du parasitisme par des strongles gastrointestinaux chez les chèvres laitières dans le SudEst de la France. INRA Prod. Anim., 12, 377-389.

Hoste H., Chartier C., Le Frileux Y., 2002. Control of gastro-intestinal parasitism with nematodes in dairy goats by treating the host category at risk. Vet. Res., 33, 531-546.

Hoste H., Guitard J.P., Pons J.C., 2003. Pâturage mixte entre ovins et bovins : intérêt dans la gestion des strongyloses gastro-intestinales. Fourrages, 176, 425-436.

Hoste H., Jackson F., Athanasiadou S., Thamsborg S., Hoskin S.O., 2006. The effects of tannin rich plants on parasitic nematodes in ruminants. Trends Parasitol., 22, 253-261.

Jain R.C., Sachdev K.N., 1995. A note on hypoglycaemic action of onion in diabetes. Curr. Med. Pract., 15, 901-902.

Kaplan R.M., 2004. Drug resistance in nematodes of veterinary importance: a status report Trends Parasitol., 20, 477-481.

Lamm D.L., Riggs D.R., 2000. The potential application of Allium sativum (garlic) for the treatment of bladder cancer. The Urologic Clinics of North America, 27, 157-162.

Lange K.C., Olcott D.D., Miller J.E., Mosjidis J.A., Terrill T.H., Burke J.M., Kearney M.T., 2006. Effect of sericea lespedeza (Lespedeza cuneata) fed as hay, on natural and experimental Haemonchus contortus infections in lambs. Vet. Parasitol., 141, 273-278.

Larsen M., 2000. Prospects for controlling animal parasitic nematodes by predacious fungi. Parasitology, 120, S121-S131.

Le Frileux Y., Napoleone M., Foubert C. Hoste H., 2006. Gestion du parasitisme. Guide pratique pour la conduite du pâturage caprin. Institut de l'Elevage (Ed), 80-113.

Leclerc H., 1922. Les Vermifuges. Précis de phytothérapie. Masson et Cie (Ed), Paris, France, 23-36.

Lumaret J.P., Errouissi F., 2002. Use of anthelmintics in herbivores and evaluation of risks for the non target fauna of pastures. Vet. Res., 33, 547-562.

Lund V., Algers B., 2003. Research on animal health and welfare in organic farming - a literature review. Livest. Prod. Sci., 80, 55-68.

Mage C., Grosmond G., 2009. Gestion des parasites gastro-intestinaux de l'agneau et des bovins adultes à l'aide d'huiles essentielles. Sem. Vét., Sous presse.

Mahieu M., Aumont G., Michaux Y. Alexandre G., Archimede H., Boval M., Theriez M. 1997. L'association d'ovins et de bovins sur prairies irriguées en Martinique. INRA Prod. Anim., 10, 55-65.

Marley C.L., Cook R., Keatinge R., Barrett J., Lampkin N.H., 2003. The effect of birdsfoot trefoil (Lotus corniculatus) and chicory (Chicorium intybus) on parasite intensities and performance of lambs naturally infected with helminth parasites. Vet. Parasitol., 112, 147-155.

McEwan J.C., Mason P., Baker R.L., Clarke J.N. Hickey S.M., Turner K., 1992. Effect of selection for productive traits on internal parasite resistance in sheep. Proc. N. Z. Soc. Anim. Prod., 52, 53-56.

Michel J.F., 1976. The epidemiology and control of some nematode infections in grazing animals. Adv. Parasitol., 14, 355-397.

Niezen J.H., Charleston W.A.G., Hodgson J., Mackay A.D., Leathwick D.M., 1996. Controlling internal parasites in grazing ruminants without recourse to anthelmintics: approaches, experiences and prospects. Int. J. Parasitol., 26, 983-992.

Osoro K., Benito-Peña A., Frutos P., Garcia P.M., Ortega-Mora L.M., Celaya R., Ferre I., 2007. The effect of heather supplementation on gastro-intestinal nematode infections and performance in Cashmere and local Celtiberic goats on pasture. Small Rum. Res., 67, 184-191.

Paolini V., Fouraste I., Hoste H., 2004. In vitro effects of three woody plant and sainfoin on third-stage larvae and adult worms of three gastro-intestinal nematodes. Parasitology, 129, 69-77.

Paolini V., De La Farge F., Prevot F. Dorchies Ph., Hoste H., 2005. Effects of the repeated distribution of sainfoin hay on the resistance and the resilience of goats naturally infected with gastrointestinal nematodes Vet. Parasitol., 127, 277-283.

Paraud C., Hoste H., Lefrileux Y., Pommare A., Paolini V, Pors I, Chartier C., 2005. Administration of Duddingtonia flagrans chlamydiospores to goats to control gastro-intestinal nematodes: dose trials. Vet. Res., 36, 157-166.

Paraud C., Lumaret J.P., Chartier C., 2007 Lack of effect of the nematophagous fungus Duddingtonia flagrans on the development of the dung beetle, Aphodius constans. Small Rum. Res., 70, 276-279.

Patout O., Devimeux J.M., 2005. La méthode HACCP appliquée à la gestion du parasitisme des agnelles. Actes de la 5 émes Journ. Techn. Pôle Scient. AB Massif Central, Roanne Chervé, France.

Pomroy W.E., 2006. Anthelmintic resistance in New-Zealand: a perspective on recent findings and options for the future. N. Z. Vet. J., 54 , 265-270.

Règlement CEE $\mathrm{n}^{\circ}$ 889/2008 de la Commission du 5 septembre 2008 portant modalités d'application du règlement $\mathrm{CE}$ 
$n^{\circ} 834 / 2007$ du Conseil relatif à la production biologique et à l'étiquetage des produits biologiques en ce qui concerne la production biologique, l'étiquetage et les contrôles. Journal Officiel de l'Union Européenne L 250 du 18 septembre 2008, 84 .

Regnault-Roger C., 2002. Biopesticides d'origine végétale. Tech et Doc, 19-39.

Saul G.R., 1996. Effects of two pasture systems on faecal nematode egg counts in breeding ewes. Aust. Vet. J., 74, 154-155.

Shaik S.A., Terrill T.H., Miller J.E., Kouakou B., Kannan G., Kaplan R.M., Burke J.M., Mosjidis J.A., 2006. Sericea lespedeza hay as a natural deworming agent against gastrointestinal nematode infection in goats. Vet. Parasitol., 139, 150-157.

Simm G., Conington J., Bishop SC, Dwyer C.M., Pattinson S., 1996. Genetic selection for extensive conditions. Appl. Anim. Behav. Sci., 49, 47-59.

Southcott W.H., Barger I.A., 1975. Control of nematode parasites by grazing management. II decontamination of sheep and cattle pastures by varying periods of grazing with the alternate host. Int. J. Parasitol., 5, 45-48.

Smith W.D., Zarlenga D.S., 2006 Development and hurdles in generating vaccines for controlling helminth parasites of grazing ruminants. Vet. Parasitol., 139, 347-359.

Stear M.J., Murray M., 1994. Genetic resistance to parasitic disease, particularly of resistance in ruminants to gastrointestinal nematodes. Vet. Parasitol., 54 , 161-176.
Steel J.W., 2003. Effects of protein supplementation of young sheep on resistance development and resilience to parasite nematode. Aust. J. Exp. Agric., 43, 1469-1476.

Tabel J., Sauvé C., Cortet J., Tournadre H., Thomas Y., Cabaret J., 2008. Fonder l'évaluation de la thérapeutique sur l'individu ou sur le groupe ? Un exemple : homéopathie et strongles digestifs des ovins. Innovations Agronomiques, 4, sous presse. http://www. inra.fr/ciag/revue innovations agronomiques

Thamsborg S.M., Roepstorff A., Larsen M., 1999. Integrated and biological control of parasites in organic and conventional production systems. Vet. Parasitol., 84, 169-186.

Torres-Acosta J.F.J., Jacobs D., AguilarCaballero A.J., Sandoval-Castro C., MayMartínez M., Cob-Galera L.A., 2004. The effect of supplementary feeding on the resilience and resistance of browsing Criollo kids against natural gastrointestinal nematode infections during the rainy season in tropical Mexico. Vet. Parasitol., 124, 217-238.

Torres-Acosta J.F.J., Jacobs D.E., AguilarCaballero A.J., Sandoval-Castro C., Cob-Galera L., May-Martínez M., 2006. Improving resilience against natural gastrointestinal nematode infections in browsing kids during the dry season in tropical Mexico. Vet. Parasitol., 135, 163-173.

Vagenas D., Jackson F., Russel A.J.F., Merchant M., Wright I.A., Bishop S.C., 2002. Genetic control of resistance to gastro-intestinal parasites in crossbred cashmere-producing goats: responses to selection, genetic parameters and relationships with production traits. Anim. Sci., 74, 199-208.

Van Wyk J.A., Bath G.F., 2002. The FAMACHA system for managing haemonchosis in sheep and goats by clinically identifying individual animals for treatments. Vet. Res., 33, 437-640.

Waller P.J., Thamsborg S.M., 2004. Nematode control in «green» ruminant production systems. Trends Parasitol., 20, 493-497.

Waller P.J., 2006a. From discovery to development: current industry perspectives for the development of novel methods of helminth control in livestock. Vet. Parasitol., 139, 1-14.

Waller P., 2006b. Sustainable nematode parasite control strategies for ruminant livestock by grazing management and biological control. Anim. Feed Sci. Technol., 126, 277-289.

Woolaston R.R., Baker R.L., 1996. Prospects of breeding small ruminants for resistance to internal parasites. Int. J. Parasitol., 26, 845-855.

Youdim K.A., Deans S.G., 2000. Effect of thyme oil and thymol dietary supplementation on the anti oxydant status and fatty acid composition of the ageing rat brain. Br. J. Nutr., 83, $87-$ 93.

Younie D., Thamsborg S.T., Ambrosini F., Roderick S., 2004. Grassland management and parasite control. In: Animal health and welfare in organic agriculture. Vaarst M., Roderick S., Lund V., Lockeretz W. (Eds), Cabi publishing, Wallingford, Oxon, UK, 227-252.

\section{Résumé}

En termes de gestion sanitaire des troupeaux, les recommandations du règlement de la commission européenne du 5 septembre 2008 (CE 889/2008), remplaçant le REPAB, visent à restreindre le recours aux traitements chimiques pour gérer les pathologies. Elles privilégient en contre-partie les règles d'hygiène et le développement de la réponse immune de l'animal. Ces principes généraux se retrouvent et sont illustrés dans la gestion du parasitisme helminthique par les strongles gastro-intestinaux qui constitue une des dominantes pathologiques de l'élevage des ruminants en systèmes $\mathrm{AB}$ et conventionnel au pâturage. Cette démarche a conduit à développer (ou à redécouvrir) des solutions techniques. Elles visent à fournir les bases agronomiques pour limiter le parasitisme par une gestion raisonnée du pâturage, à stimuler la réponse de l'hôte par divers moyens, à évaluer l'efficacité de thérapeutiques naturelles ou alternatives (homéopathie, aromathérapie, phytothérapie et nutricament) et enfin à proposer de nouveaux concepts d'application des anthelminthiques de synthèse. A terme, cette démarche analytique devrait fournir aux éleveurs une «offre de solutions» à adapter aux diverses situations afin d'aboutir à une approche intégrée du parasitisme, compatible avec les principes de l'agriculture durable.

\section{Abstract}

\section{Alternatives to chemical anthelmintics in organic farming}

The objectives are the following with regards to the management of herd and flock health in the Organic farming system: i) to restrict the use of chemical drugs and in contrast ii) to favour the application of hygienic rules and iii) to stimulate the development of an effective host response against pathogens. These general principles also have to be applied to control parasitic infections. They are illustrated by application to the management of nematodes of the gastro intestinal tract in ruminants, these parasitic diseases being a major worldwide constraint in the breeding of ruminants. Control solutions are currently being explored or rediscovered. They aim at i) providing the agronomical bases to helminths control by a rational management of pastures; ii) stimulating the host immune response through different means; iii) evaluating the efficacy of new drugs (i.e. phytotherapy, aromatherapy, homeopathy and nutraceuticals) and iv) proposing a new concept of application of chemical anthelmintics. The perspectives are to provide to organic and conventional farmers, a «basket of options» to be adapted to the various situations, in order to achieve a more sustainable integrated approach of parasite control in ruminants.

HOSTE H., CABARET J., GROSMOND G., GUITARD J.-P., 2009. Alternatives aux traitements anthelminthiques en élevage biologique des ruminants. Inra Prod. Anim., 22, 245-254. 